\title{
Thermoregulation Mediated by Conditioned Heart-Rate Changes in Pigeons*
}

\author{
Markus Sieland, Juan D. Delius, Werner Rautenberg, and Bärbel May \\ AG Experimentelle Tierpsychologie, Psychologisches Institut and AG Temperaturregulation, Abteilung Biologie, \\ Ruhr-Universität, D-4630 Bochum, Federal Republic of Germany
}

\begin{abstract}
Summary. Pigeons were subjected to an apparent heat $\left(44{ }^{\circ} \mathrm{C}\right)$ or cold $\left(30^{\circ} \mathrm{C}\right)$ load by means of a thermode located next to their thermosensitive spinal cord. They were then able to obtain brief thermonormality $\left(40^{\circ} \mathrm{C}\right)$ spells if they changed their heart-rate by a preset amount above (or below) their baseline heartrate. In the final experiments the animals increased (or decreased) their heart-rate by about $25 \%$ within 12 training sessions, thereby achieving a partial thermoregulation: they were able to maintain their spinal temperature at a normal level for about $25 \%$ of the time. Extinction sessions, in which the heart-rate changes did not have any thermoregulatory effect, and control sessions, in which thermonormality periods were given independently of the heart activity, yielded baseline heart-rates. It is concluded that the cardiac activity related to thermoregulation may be subject to modification through learning and thus may not differ fundamentally in this respect from some homeothermic behavioural responses.
\end{abstract}

\section{Introduction}

Birds, like many other animals, have available two types of effector mechanisms to regulate body temperature: behavioural and autonomic. It has generally been assumed that the former type of responses are largely learned and indeed it has recently been shown that some more or less arbitrary behaviour patterns of birds can be conditioned to play a role in thermoregulation (Budgell 1971; Schmidt and Rautenberg 1975; Laudenslager and Hammel 1977; Horowitz et al. 1978; Schmidt 1978 a; Schmidt and Simon 1979; Rautenberg et al. 1980). Autonomic homoeothermic responses, on the other hand, have been considered to be largely unmodifiable by experience, that is of

\footnotetext{
* Offprint requests should be sent to: J.D. Delius, Psychologisches Institut, Ruhr-Universität, Universitätsstrasse 150, D-4630 Bochum, Federal Republic of Germany
}

innate origin. In this paper we report experiments that suggest that this notion may be too extreme in that at least the cardiac activity of pigeons in a thermoregulatory setting can be easily altered by instrumental conditioning.

The general design of the experiments was to place pigeons under an apparent thermal load by locally heating (or cooling in some experiments) their thermosensitive spinal cord (Rautenberg 1969; Rautenberg et al. 1972; Necker 1975; Schmidt 1978b) and allowing them a brief relief from this load whenever they increased (or decreased) their heart frequency to a set rate over a determined period of time above (or below) their typical baseline rate. A specified cardiac response was thus reinforced by the brief omission of an aversive motivating thermal stimulus complying with the typical instrumental or operant conditioning paradigm (Mackintosh 1974). We advisedly speak of an apparent thermal load because in terms of calories the thermal stress applied was really relatively insignificant. The heart-rate was chosen as the operant response mainly because of its easy quantification and its undoubted involvement in normal thermoregulation (Smith 1972; Petran 1972; Bech et al. 1980).

\section{Materials and Methods}

Six adult homing pigeons (Columba livia) of local origin were used. While the pigeons were anaesthetized with pentobarbital $(3 \mathrm{mg} /$ $100 \mathrm{~g}$ body-weight, intramuscular) the dorsal surface of the 5th thoracic vertebra was exposed. The bone was trephinated and a U-shaped length of polythene tubing of $0.6 \mathrm{~mm}$ outer diameter was inserted cephalad into the peridural space until the tip of this thermode reached the anterior cervical vertebrae. A blindending length of polythene tubing of $1.0 \mathrm{~mm}$ outer diameter that later was to take up a thermocouple was inserted between the two legs of the thermode until it reached the 2 nd thoracic vertebra. The wound was carefully closed with sutures, with the three ends of tubing projecting some $15 \mathrm{~mm}$ out of the skin.

Four stainless steel wire electrodes (each consisting of three 


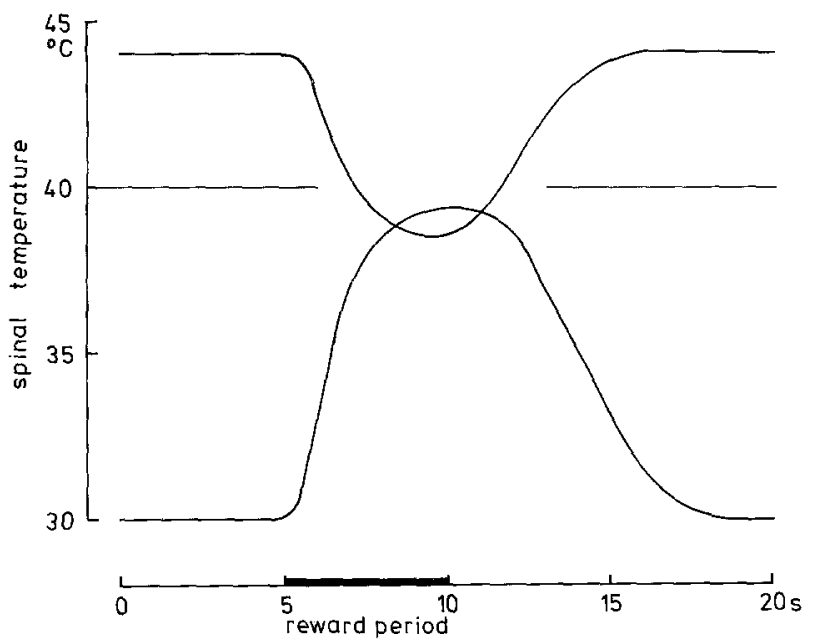

Fig. 1. Spinal temperature course during the reward periods for both the heat $\left(44^{\circ} \mathrm{C}\right)$ and the cold load $\left(30^{\circ} \mathrm{C}\right)$ conditions. The normal spinal temperature late in the evening $\left(40^{\circ} \mathrm{C}\right)$ is indicated. Based on 6 sets of measurements on pigeons $E$ and F. All measurements fell within $\pm 0.5^{\circ} \mathrm{C}$ of the traces shown

strands of $0.1 \mathrm{~mm}$ diameter) were drawn under the two scapular bones and the two pubic spines with the help of a thick, curved hypodermic cannula. The electrode ends protruding out of the skin were threaded through four lengths of insulating tubing, brought together on the back of the subject and terminated in a four-pole miniature socket. Animals were allowed at least one week recovery after the operation before the experiments began.

The subjects were restrained with a corset and placed in a foam rubber bed within a sound attenuating chamber. The ECG electrodes (left scapular, right pubic) were connected through to an amplifier-filter followed by a trigger stage set to give a standard logic pulse to each heart-beat. For ease of adjustment the ECG waves and the pulses were displayed on separate channels of an oscilloscope. The pulses fed into a digital programming system described below.

The spinal thermode inlet was linked via a short length of plastic tubing and a branch to the outlets of a pair of electromagnetic valves. These could be switched by the digital programming system so that either water at a temperature leading to a thermal load or water at a temperature driving towards normal body temperature passed through the thermode. Water at the required temperature and pressure was fed into the inlets of the electromagnetic valves from two separate thermostats with high pressure circulating pumps. The bypass outlets of the two valves were fed back to the corresponding thermostats. The outlet of the thermode was lead to a waste drain. The flow rate was kept constant about $10 \mathrm{~m} 1 / \mathrm{min}$.

A thermocouple was inserted into the spinal guide tube and connected to a digital thermometer, its reference junction being immersed in an ice-bath fitted with a mercury thermometer. The waterbath temperatures were always adjusted so that the spinal cord was heated to $44 \pm 0.5^{\circ} \mathrm{C}$ (or in some experiments cooled to $30 \pm 0.5^{\circ} \mathrm{C}$ ). Due to the convective losses in the conduit system this required an actual waterbath temperature of $75 \pm 5^{\circ} \mathrm{C}$ (or $10 \pm 5^{\circ} \mathrm{C}$ ). The resting pigeon's typical spinal cord temperature at early night, when most experiments took place, is $40 \pm 0.5^{\circ} \mathrm{C}$ (Graf and Necker 1979). In order to bring the spinal temperature down (or up), close to this resting temperature, within the $5 \mathrm{~s}$ reward period, it was necessary to set the corresponding water-bath temperature at $30 \pm 5^{\circ} \mathrm{C}$ ( or $60 \pm 5^{\circ} \mathrm{C}$ ). The heat load temperature was limited by the need to avoid thermal injury to the spinal cord. The cold load temperature, not constrained by such consider- ations, was intended to maximise the motivating effect, although in retrospect it may have been somewhat extreme (Görke 1980). While the cooling reward overshot slightly the normal spinal cord temperature, the heating reward for technical reasons did not quite reach that temperature. Figure 1 shows the spinal temperature course obtained around the reward periods under both conditions. The ambient temperature in the experimental chamber was $20 \pm$ $1{ }^{\circ} \mathrm{C}$.

The digital programming system was arranged such that each of two presettable counters registered the heart-beat pulses within overlapping $10 \mathrm{~s}$ periods, each resetting automatically at the end of the corresponding periods. If the count on either of the counters exceeded (or, in some experiments, fell short of) the criterion number of beats per $10 \mathrm{~s}$ that had been manually preset on both counters, the system switched the electromagnetic valves into the reward issuing position for $5 \mathrm{~s}$. Simultaneously a potential further reward at the end of the now ongoing counting period was prevented. This avoided that the thermal change induced by the ongoing reward might, through some circulatory reflex mechanism, lead automatically to an additional reward. The chamber illumination was dimmed (or brightened in some experiments) for the reward period, but this brightness change could be stepwise diminished with a rheostat, until absent. The rewards delivered were registered on a separate counter.

The heart-beat pulses were also fed into an instantaneous ratemeter whose output was written out continuously with a penrecorder. This trace was periodically calibrated with a precision pulse generator. Another channel of the recorder registered the reward periods. Changes in the preset criterion were also marked on the recorder chart.

On every second day, weekends included, subjects were each given a $90 \pm 10 \mathrm{~min}$ session beginning approximately at $20.00 \mathrm{~h}$ when the laboratory was quiet. The first sessions were adapting sessions in which the baseline heart-rate under continuous thermal load was established for each pigeon. Six sessions of training followed. If the animals were expected to learn to produce a tachycardic response for the thermoregulatory reward, the first step was to preset the heartrate counters 2 beats $/ 10 \mathrm{~s}$ above the mean baseline rate established in the two preceding baseline sessions. If the subject managed to obtain more than 5 rewards in the final $5 \mathrm{~min}$ of a $15 \mathrm{~min}$ period then the criterion rate was increased by another 2 beats $/ 10 \mathrm{~s}$ and so forth. If the animal, having been given an increased criterion, did not achieve 5 rewards in the last $5 \mathrm{~min}$ of the following $15 \mathrm{~min}$ period the criterion rate was decreased until the reward frequencies increased again above the 5 rewards $/ 5$ min standard. The overall aim was to maintain a reward frequency of between 5 and 10 per $5 \mathrm{~min}$, all the while attempting to increase the criterion heart-rate. The initial heart-rate criterion of the next sessions was set 4 to 6 beats per $10 \mathrm{~s}$ below the terminal one used in the preceding session. During the last three training sessions the light signal accompanying the rewards was stepwise decreased, so that from the first of the 6 maintenance sessions onwards there was no light signal accompanying the thermal rewards.

In the first three sessions of the maintenance block the procedure was the same as in the training sessions except that by and large no attempt was made to increase the criterion rate beyond the maximum achieved during the training block. During the last three sessions of the maintenance block the criterion rates were kept largely constant, only when the animal persistently exceeded the 5 rewards $/ 5$ min standard was it increased.

During the 6 extinction sessions that followed no rewards at all were issued, thus also no criterion heart-rates were set. During 2 control sessions rewards were again given but independently of the current cardiac rate of the subjects. Rather, they were issued in the same temporal sequence as during the last two maintenance sessions of the same subject. 

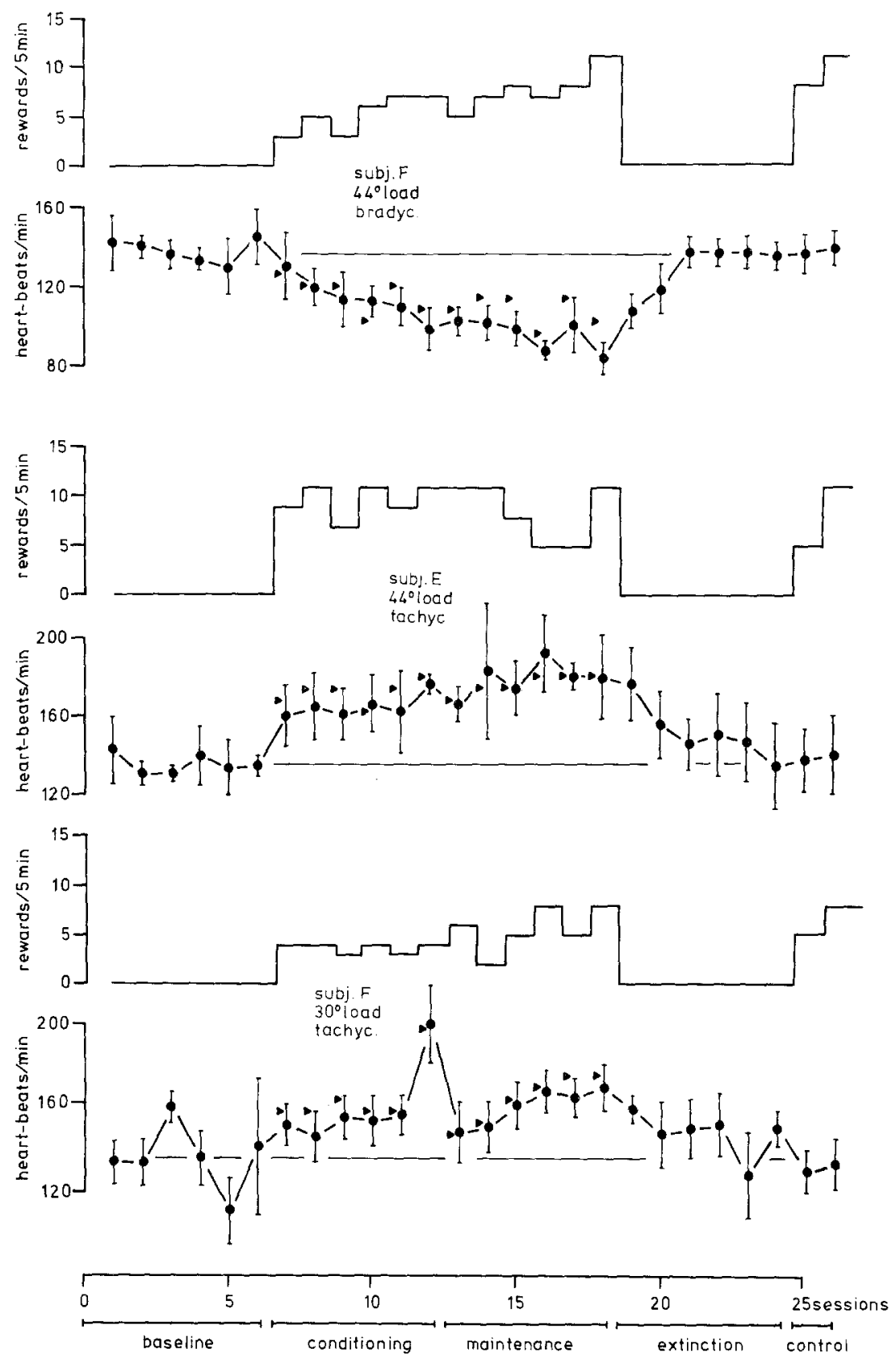

Fig. 2. Heart-rate courses during the three types of experiments, typical examples. Mean session heart-rates with standard deviations (based on 5 min sampling). Triangles: mean criterion heart-rate for each conditioning and maintenance session. Horizontal lines: average heartrate during baseline sessions. Step functions: mean reward rate obtained during the various sessions

In those experiments where the response demanded was bradycardia, the procedure was analogous except that the subject's heartrate had to fall short of a preset rate for a reward to be delivered. The preset criterion was of course set at lower and lower rates as the training progressed but otherwise the same strategy as just described was employed.

\section{Results}

Nine experiments, three of them with a still somewhat preliminary design and procedure, were completed. Five of them were carried out with the subject's spinal cord being heated $\left(44^{\circ} \mathrm{C}\right)$ and receiving thermonormality rewards for tachycardic responses. Two experiments similarly involved spinal heating but the re- sponse demanded was bradycardia. Finally two experiments involved spinal cooling $\left(30^{\circ} \mathrm{C}\right)$ and the animals being reinforced with thermonormality for tachycardic responses.

For evaluation the experimental sessions were divided into consecutive $5 \mathrm{~min}$ periods. The number of reinforcements was counted, the heart-rate at the end of each period was noted and the criterion rate operating during the period was recorded. For inspection these values were plotted, separately for each session, as a function of the sequence of $5 \mathrm{~min}$ periods. Mean values of these measurements for each session were calculated. Additionally the standard deviations of the heart-rate scores were computed. These 


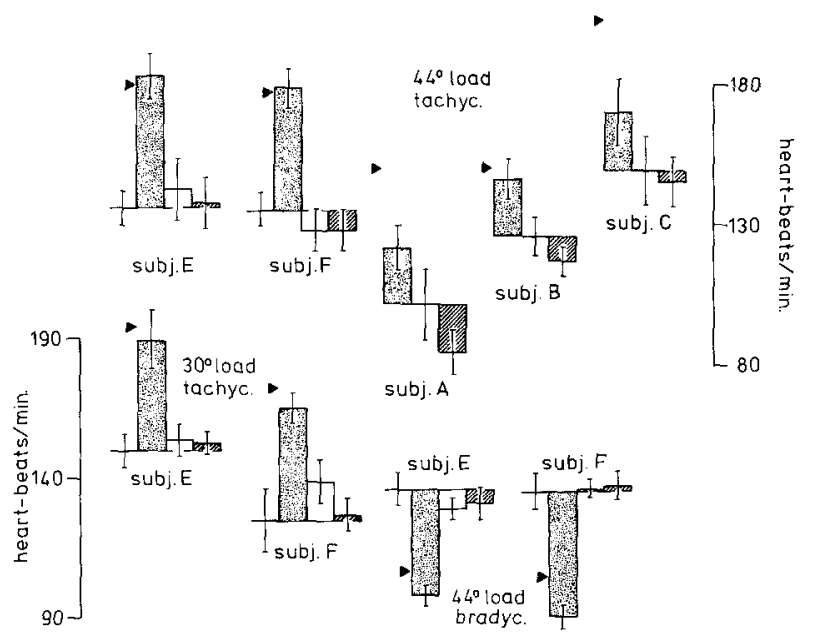

Fig. 3. Summary diagram of results for the 9 experiments, showing average heart-rates and standard deviations. The 4 segments of each graph represent results during the last 3 baseline sessions (this initial segment is missing for 3 preliminary experiments involving subjects $\mathrm{A}, \mathrm{B}$ and $\mathrm{C}$ ), during the last 3 maintenance sessions (dotted columns), during the last 3 extinction sessions (white columns, or middle segments for subjects A, B and C) and during the 2 yoked control sessions (shaded columns). Triangles: average criterion rate operating during the relevant maintenance sessions. As there were no baseline sessions during the preliminary experiments, the extinction sessions are taken as reference

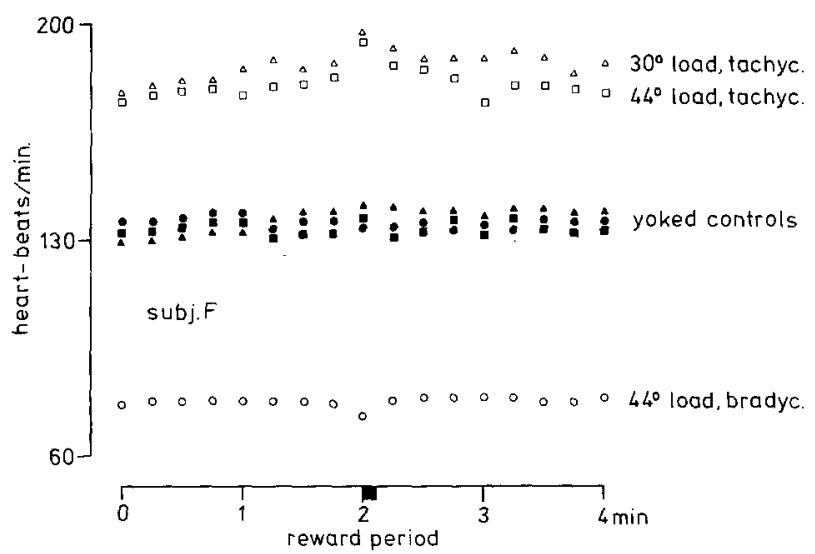

Fig. 4. Average peri-reward heart-rates of pigeon $F$ during the last 20 rewards of both the final maintenance sessions (open symbols) and the final control sessions (filled symbols) during the 3 types of experiments (triangles, squares, circles)

data were graphed as a function of the sequence of sessions, separately for each subject and experiment. Figure 2 presents representative examples for the three experimental conditions.

In each experiment the pigeons modified their heart-rate in the sense of the conditioning paradigm (Fig. 3). The overall increase (or decrease) in heartrate of between 17 and $43 \%$ in the last three sessions of the maintenance block as compared with the heartrate in the last three baseline sessions or the three final extinction sessions and in the two control sessions was in each case highly significant (Mann-Whitney, $P<0.01$ or better).
To assess in more detail the conditioned cardiac behaviour we computed separate peri-reward average heart-rate courses for the last maintenance and the control sessions of the six final experiments. The heart-rate was sampled every $15 \mathrm{~s}$ for $2 \mathrm{~min}$ before, and 2 min after, the onset of the last 20 reinforcement periods. Since the results for the two birds involved are similar, Fig. 4 shows only those of one of them. It is obvious that in the maintenance sessions the rewards were not obtained by steep transient heartrate changes but rather by slight, gradual modifications of stably high (or low) rates. This, however, may be due to the particular strategy used to set the criterion rates in these experiments since in some of the preliminary experiments, where the criteria changes were often more abrupt, the pigeons undoubtedly obtained the rewards with burst-like cardioaccelerations. It is important to note again that the pseudo-rewards during the control sessions did not have any appreciable effects on the course of the heart-rate.

\section{Discussion}

There are two grounds for arguing that, in each case, the heart-rate response must have been a reaction acquired through conditioning. Firstly, under extinction, that is in the absence of a thermoregulatory consequence, the heart-rate strove towards the preconditioning baseline. Secondly, and perhaps more importantly, the heart-rate did not depart from the baseline rate under control conditions where the animals received thermal 'rewards' dissociated from their cardiac activity. This latter control condition ensured that changes in heart-rate seen during the maintenance period were not per se consequences of the thermal change associated with reward. This possibility is further excluded by the fact that the same pigeons could be alternately trained to produce tachycardia or bradycardia in the same thermoregulatory setting, involving an overheated spinal cord with cooling as reward, and, moreover, the same animals could be taught a tachycardic response for a thermoregulatory heating of an undercooled cord.

How the animals brought about the instrumental heart-rate responses must remain uncertain until further work has been completed. It can not be excluded that what they learned was a manoeuvre involving some skeletal or visceral change in activity that indirectly, through cardiovascular regulation reflexes, released the required heart-frequency changes. Panting, shivering or escape efforts are examples of peripheral responses which could be expected to cause such secondary heart-rate changes. To exclude them it would be necessary to experiment with curarized subjects (Miller and Dworkin 1974; see Migani-Wall et al. 1977 for techniques). This has in fact been done by 
Cabanac and Serres (1976) who carried out experiments analogous to ours using rats and found that paralysed animals were still capable of learning thermoregulatory heart-rate responses. Of course, curarization does not exclude the possibility that the collateral autonomic activation, known to normally accompany neural motor outflow to the skeletal musculature (Cohen and Obrist 1975), may be involved. Regarding our animals we can only report that no consistent skeletal responses associated with the delivery of reward could be observed. The animals maintained a generally passive, relaxed attitude throughout. In particular they showed little or no visible panting or shivering although these responses are known to be released by spinal heating and cooling respectively (Rautenberg et al. 1972). We suspect that the semichronic thermal load stimulation might have led to an habituation of these responses. The thermal stimulation associated with rewards was probably too brief to be effective. In future work this issue will merit closer examination. The most pressing question, however, is to find out whether the thermoregulatory setting of such responses as shivering and panting can be modified by conditioning paradigms.

Our experiments suggest that it may not be valid to assume that the autonomic thermoregulatory responses of birds are innate and unmodifiable but that rather they may become established and be maintained through conditioning, not unlike what is thought to be the case with some behavioural thermoregulatory reactions. In this context it may be useful to keep in mind that the cardiac activity of birds is easily modified through classical, Pavlovian conditioning (see, for example, Delius and Tarpy 1974; Cohen and Goff 1978), although this has not yet been demonstrated in a thermoregulatory context. Learning processes could be useful in bringing about the optimal integration of behavioural and autonomic thermoregulation mechanisms (Schmidt 1978a; Schmidt and Simon 1979) where other organismic needs than homoeothermy may also have to be considered in as far as they compete for effector control (see Rautenberg et al. 1980; Gabrielsen et al. 1977; Gabrielsen and Steen 1979).

This work was supported by the Interdisciplinary Research Fund of the Ruhr-Universität and the Deutsche Forschungsgemeinschaft through its Sonderforschungsbereich 114. We thank Dr. I. Schmidt (Bad Nauheim) for much advice and discussion and Dr. J. Emmerton, Miss A. Lohmann and Mrs. D. Hagenkötter for assistance of various kinds.

\section{References}

Bech C, Rautenberg W, May B, Johansen K (1980) Effect of spinal cord temperature on carotid blood flow in the peking duck. Pflügers Arch 385:269-271
Budgell P (1971) Behavioural thermoregulation in the barbary dove. Anim Behav 19:524-531

Cabanac M, Serres P (1976) Peripheral heat as a reward for heat rate response in the curarized rat. J Comp Physiol Psychol $90: 435-441$

Cohen DH, Goff DM (1978) Conditional heart-rate change in the pigeon: analysis and prediction of acquisition patterns. Physiol Psychol 6:127-141

Cohen DH, Obrist PA (1975) Interactions between behaviour and the cardiovascular system. Circ Res 37:693-706

Delius JD, Tarpy R (1974) Stimulus control of heart-rate by auditory pattern in pigeons. J Exp Anal Behav $21: 297-306$

Gabrielsen G, Steen JB (1979) Tachycardia during egg hypothermia in incubating patarmigan (Lagopus lagopus). Acta Physiol Scand 107:273-277

Gabrielsen G, Kanwisher J, Kanwisher JB (1977) Emotional bradycardia: a telemetry study on incubating willow grouse (Lagopus lagopus). Acta Physiol Scand 100:255-257

Görke K (1980) Influence of spinal cord temperature changes on reflex discharge and spontaneous activity of spinal motor neurons in pigeons and leguans. J Comp Physiol 139:251-259

Graf R, Necker R (1979) Cyclic and non-cyclic variations of spinal cord temperature related with temperature regulation in $\mathrm{pi}$ geons. Pflügers Arch 380:215-220

Horowitz KA, Scott NR, Hillman PW, van Tienhoven A (1978) Effects of feathers on instrumental thermoregulatory behaviour in chickens. Physiol Behav 21:233-238

Laudenslager ML, Hammel HT (1977) Environmental temperature selection by the chukar partridge (Alectoris chukar). Physiol Behav 19:543-548

Mackintosh NJ (1974) The Psychology of Animal Learning. Academic Press, London

Migani-Wall SA, Cabot JB, Cohen DH (1977) Development of a neuromuscular blocking procedure for heart-rate conditioning in immobilized pigeons. Psychophysiol 14:499-506

Miller NE, Dworkin BR (1974) Visceral learning: recent difficulties with curarized rats and significant problems for human research. In: Obrist PA, Black AH, Brener J, DiCara LV (eds) Cardiovascular psychophysiology. Aldine, Chicago, pp 312-331

Necker R (1975) Temperature-sensitive ascending neurons in the spinal cord of pigeons. Pflügers Arch 353:275-286

Petran M (1972) Die Wirkung allgemeiner and spinaler Hyperthermie auf Atmung, Herzfrequenz und Blutdruck der Taube. Diplom Thesis, Abt Biologie, Univ Bochum

Rautenberg W (1969) Die Bedeutung der zentralnervösen Thermosensitivität für die Temperaturregulation der Taube. Z Vergl Physiol 62:235-266

Rautenberg W, Necker R, May B (1972) Thermoregulatory responses of the pigeon to changes of the brain and the spinal cord temperatures. Pflügers Arch 338:31-42

Rautenberg W, May B, Arabin G (1980) Behavioral and autonomic temperature regulation in competition with food intake and water balance of pigeons. Pflügers Arch 384:252-260

Schmidt I (1978 a) Interactions of behavioural and autonomic thermoregulation in heat stressed pigeons. Pflügers Arch 374:47-55

Schmidt I (1978 b) Behavioral and autonomic thermoregulation. in heat stressed pigeons modified by central stimulation. J Comp Physiol 127:75-87

Schmidt I, Rautenberg W (1975) Instrumental thermoregulatory behaviour in pigeons. J Comp Physiol 101 :225-235

Schmidt I, Simon E (1975) Interaction of behavioural and autonomic thermoregulation in cold exposed pigeons. J Comp Physiol $133: 151-157$

Smith RM (1977) Circulation, respiratory volumes and temperature regulation of the pigeon in dry and humid heat. Comp Biochem Physiol [A] 43: 477-490 